\title{
Vólvulo gástrico: reporte de caso en el Hospital Central Universitario del Estado de Chihuahua
}

\author{
Gastric volvulus: case report in Central Hospital of the State of Chihuahua \\ Enrique Villela Cañas, ${ }^{*}$ Marco Javier Carrillo Gorena, ${ }^{*}$ \\ Armando Soto Pérez, ${ }^{*}$ David Alfonso Aguirre Baca, ${ }^{*}$ Luis Bernardo Enríquez Sánchez, \\ José Guadalupe Padilla López, ${ }^{*}$ Armando Isaac Reyes Carrillo, ${ }^{*}$ \\ Manuel David Pérez Ruíz, ${ }^{*}$ Arely Estefania Contreras Pacheco*
}

Palabras clave: Vólvulo gástrico, hernia hiatal, isquemia gástrica, gastrectomía.

Keywords: Stomach volvulus, hiatal hernia, gastric ischemia, gastrectomy.
${ }^{*}$ Departamento de Cirugía General del Hospital Central Universitario "Dr. Jesús Enrique Grajeda Herrera", Chihuahua, México.

Recibido: 20/02/2020 Aceptado: 23/03/2021

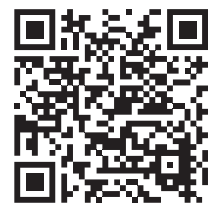

RESUMEN

El vólvulo gástrico es una entidad clínica poco común, caracterizada por una rotación anormal del estómago en uno de sus ejes (longitudinal o axial), entidad con elevada morbimortalidad y riesgo de complicaciones (obstrucción o perforación). Es necesario tener alta sospecha clínica para diagnosticarlo tempranamente y tratarlo. Reporte de caso de vólvulo gástrico en mujer de 50 años que ingresa por cuadro abdominal de tres días de evolución con dolor, náusea y vómito. Durante laparotomía exploradora se encuentra hernia hiatal grado IV con vólvulo gástrico asociado, se trata quirúrgicamente; sin embargo, tiene una evolución tórpida y fallece por complicaciones.

\section{ABSTRACT}

Gastric volvulus is a rare clinical entity characterized by an abnormal rotation of the stomach in one of its axes (longitudinal or axial) which has a high morbidity/ mortality and high risk of complications (obstruction or perforation). It is necessary to have a clinical suspicious in order to give an early diagnosis and prevent complications. Report of a case of a 50-year-old female admitted for a 3-day history of abdominal pain, nausea and vomiting. By laparotomy we found a hiatal hernia grade IV and gastric volvulus. It is treated surgically; however, it has a torpid evolution and dies from complications.

\section{INTRODUCCIÓN}

E I vólvulo gástrico (VG) (del latín volvere: vuelta) es una condición poco común definida como una rotación anormal del estómago sobre su eje a más de $180^{\circ}{ }^{1}$ descrita inicialmente en 1866 por Berti. ${ }^{2}$ El retraso en el diagnóstico resulta en tasas altas de morbilidad y mortalidad debido a sus complicaciones potenciales, por lo que suele requerir un tratamiento quirúrgico urgente. $^{3}$ En una revisión de la bibliografía realizada en la base de datos de PubMed de 1999-2018 se buscó información sobre la presentación clínica, el tipo de VG, su etiología, las pruebas diagnósticas, el tratamiento y resultados en donde los casos que no presentaran dicha información fueron excluidos, resultó únicamente en 43 casos reportados. El
VG mesenteroaxial (51.1\%) fue el más frecuente, seguido del órgano-axial (46.5\%). El tratamiento quirúrgico fue el pilar terapéutico en $90.7 \%$ de los pacientes. Se reportaron tres defunciones y el resto de los pacientes con recuperación exitosa, ${ }^{2}$ en otros reportes hasta el 2009 se habían informado 350 casos en el ámbito mundial. ${ }^{4}$ Es una patología con una mayor frecuencia en la edad pediátrica $(20 \%) .{ }^{4}$ Mientras que el pico de incidencia en los adultos es alrededor de la quinta década de la vida. ${ }^{4}$ La mortalidad con un diagnóstico y tratamiento oportuno de VG agudo es de $15-25 \%$ y en VG crónico de $0.13 \%{ }^{2}$ sin embargo, con un tratamiento tardío o complicaciones se reporta una tasa de $30-50 \% .{ }^{5}$ Puede ser clasificado de acuerdo con su etiología y eje de rotación. En los pacientes pediátricos, las causas

Citar como: Villela CE, Carrillo GMJ, Soto PA, Aguirre BDA, Enríquez SLB, Padilla LJG, et al. Vólvulo gástrico: reporte de caso en el Hospital Central Universitario del Estado de Chihuahua. Cir Gen. 2020; 42 (4): 306-310. https://dx.doi. org/10.35366/101400 
primarias predominan debido a inmadurez del estómago y sus ligamentos o por defectos herniarios diafragmáticos congénitos; ${ }^{6}$ mientras que en los adultos el mayor porcentaje son secundarios, ${ }^{5}$ debido a alteraciones anatómicas del estómago o de los órganos que lo rodean, la causa más común es la hernia paraesofágica.

\section{PRESENTACIÓN DEL CASO}

Ingresa mujer de 50 años, con diabetes tipo 2 e hipertensión arterial sin tratamiento, obesidad mórbida e histerectomía hace 20 años como antecedentes relevantes. Inició su padecimiento tres días previos a su llegada con dolor tipo cólico en epigastrio, progresivo, distensión abdominal, náusea y vómitos en múltiples ocasiones, disnea en reposo, episodios febriles no cuantificados y cinco días sin evacuar. Se registran signos vitales: tensión arterial 133/100 mmHg, frecuencia cardiaca 122 latidos por minuto, frecuencia respiratoria de 36 respiraciones por minuto, temperatura 37.7 ${ }^{\circ} \mathrm{C}$. En la exploración, los campos pulmonares izquierdos estaban hipoventilados, abdomen rígido con distensión generalizada, timpánico a la percusión y dolor intenso a la palpación de predominio en epigastrio, peristalsis ausente y rebote positivo. Se inició su reanimación hídrica y se solicitaron estudios de gabinete. Con ayuda de la tomografía axial computarizada se integró un diagnóstico preoperatorio de hernia hiatal grado IV y megacolon tóxico (Figura 1). En los resultados de laboratorio: leucocitos de 16,300 células/ $\mu$ l, neutrófilos 14,000 células/ $\mu \mathrm{l}$, hemoglobina 17.3 $\mathrm{g} / \mathrm{dl}$, plaquetas 409,000 u/ $\mathrm{\mu l}$, gasometría arterial
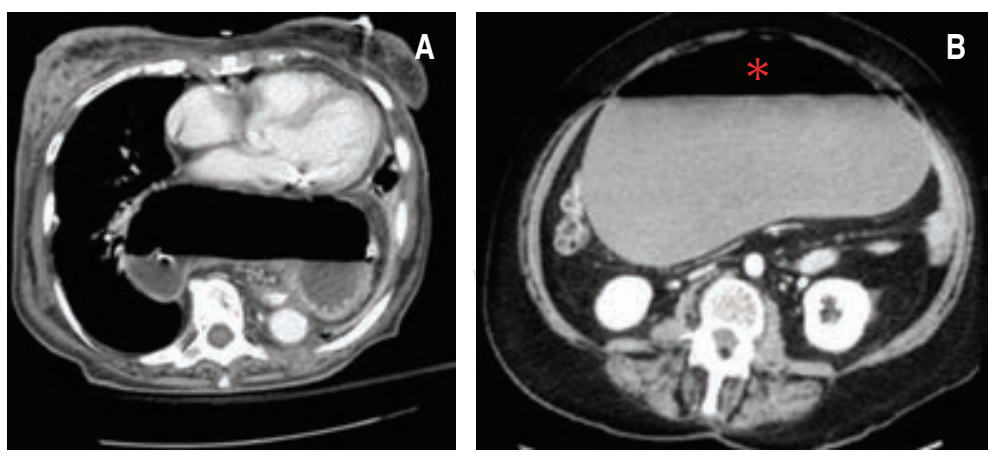

Figura 1: Tomografía axial computarizada de mujer de 50 años. A) Se observa hernia hiatal con estómago intratorácico. B) Dilatación con nivel hidroaéreo presente $(*)$. con lactato de $5.5 \mathrm{mmol} / \mathrm{l}$ y $\mathrm{pH}$ 7.48. Se hizo descompresión gástrica con sonda nasogástrica (SNG) sin dificultad para su colocación y se administró ranitidina $50 \mathrm{mg}$ iv, dexametasona $8 \mathrm{mg}$ iv, metoclopramida $10 \mathrm{mg}$ iv, ceftriaxona $1 \mathrm{~g}$ iv, butilhioscina $20 \mathrm{mg}$ iv, y paracetamol $1 \mathrm{~g}$ iv. En la laparotomía exploradora (12/07/18) se encontró una $\mathrm{HH}$ grado IV con VG secundario tipo II asociado con necrosis gástrica, se hizo la reducción del saco herniario y del VG, funduplicatura tipo Nissen y gastrectomía parcial con técnica de Billroth II en omega de Brown manual, con prolene aguja intestinal 3-0 en dos planos con puntos de Connell y Mayo, así como Lembert de refuerzo y plastía de hiato esofágico con prolene aguja intestinal 2-0 puntos simples y sin complicaciones al momento; se indicó esquema antibiótico con ciprofloxacino 400 mg iv, cada 12 horas por 10 días y metronidazol 500 mg iv, cada ocho horas por 10 días y fue llevada a la Unidad de Cuidados Intensivos para su recuperación. Una semana después, presentó dehiscencia del $50 \%$ de la anastomosis gastroyeyunal, con fuga de contenido gástrico. Se realizó un cierre primario gástrico con parche de Graham y se colocó bolsa de Bogotá. En los estudios de control presentó leucocitosis de 22 mil células/ $\mu \mathrm{l}$, neutrófilos 18 mil células $/ \mu \mathrm{l}$, hemoglobina $8 \mathrm{mg} / \mathrm{dl}$, plaquetas $123 \mathrm{mil} \mathrm{u} / \mu \mathrm{l}$, pH de 7.31 , lactato de $3.9 \mathrm{mmol} / \mathrm{l}$, falla renal asociada con creatinina de $3.2 \mathrm{mg} / \mathrm{dl}$, urea de $65 \mathrm{mg} / \mathrm{dl}$, nitrógeno ureico $45 \mathrm{mg} / \mathrm{dl}$, sodio $153 \mathrm{mEq} / \mathrm{l}$, potasio $2.8 \mathrm{mEq} / \mathrm{l}$, cloro 110 $\mathrm{mEq} / \mathrm{l}$; después, y debido a la persistencia de la fuga, se hizo un aseo quirúrgico con gastrectomía parcial y anastomosis gastroyeyunal en Y de Roux en dos planos con puntos de Connell y Mayo y refuerzo de puntos Lembert con prolene aguja intestinal 3-0, posterior yeyunostomía utilizando seda 2-0 con puntos simples a aponeurosis de pared abdominal. En las siguientes semanas se sometió a cuatro aseos quirúrgicos, recambio de bolsa de Bogotá y gastrostomía tipo Stamm debido a fuga intestinal. Persistió con cuadro de sepsis abdominal, evolucionó con perforación de la anastomosis y se colocó sonda T gástrica, encontrándose la cavidad con abundante material purulento y fétido, además de abdomen congelado. Durante su estancia se manejó con antibioticoterapia múltiple con ciprofloxacino y metronidazol, cambiando a meropenem 500 mg iv, cada ocho horas y posterior ajuste a tigeciclina 


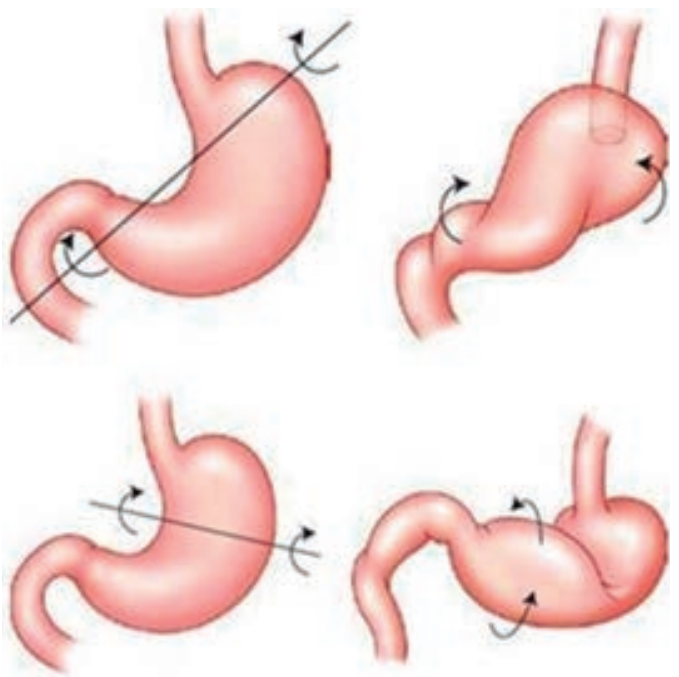

Figura 2: Parte superior: vólvulo gástrico órganoaxial, sobre eje longitudinal. Parte inferior: vólvulo gástrico mesentérico-axial con rotación sobre eje horizontal. Tomado de: Caldaro T, et al. ${ }^{3}$

100 mg iv, continuando con 50 mg iv, cada 12 horas y vancomicina $1 \mathrm{~g}$ iv, cada 12 horas por 10 días, además de nutrición parental total, sin presentar mejoría. Por cultivos de líquido peritoneal positivos a Staphylococcus aureus y Acinetobacter baumannii multirresistentes, obesidad mórbida y estado nutricional deficiente, desarrolló un choque séptico de foco abdominal, sin respuesta a medidas de reanimación, falleciendo un mes después de su ingreso.

\section{DISCUSIÓN}

El VG es una condición de baja frecuencia con una tasa alta de mortalidad (30-50\%), por lo que es necesario conocer la patología y su presentación para realizar un diagnóstico e intervención oportunos. ${ }^{2,7}$ La clasificación utilizada es la de Singleton (Figura 2):

Tipo I. Órgano-axial: rotación del estómago sobre su eje longitudinal, el cual se extiende de la unión gastroesofágica al píloro. El antro gira en sentido opuesto al fundus gástrico. Es la entidad más común (60\% de los casos).

Tipo II. Mesentérico-axial: rotación del estómago en su eje horizontal que pasa a través de las curvaturas mayor y menor. Se presenta como una condición idiopática con síntomas crónicos o intermitentes (30\% de los casos).

Tipo III. Mixto: combinación rotación órgano-axial y mesentérico-axial, es una entidad extremadamente rara ( $2 \%$ de los casos).

Tipo IV. Sin clasificar (8\%).

El primario se debe a defectos en el anclaje gástrico por la hiperlaxitud o agenesia de los ligamentos gastrocólico, gastrohepático, gastrofrénico y gastroesplénico o por alteraciones en el hiato esofágico, fijación retroperitoneal de duodeno y vasos gástricos cortos. ${ }^{8}$ El secundario $(65-70 \%$ de los casos) se debe a trastornos de anatomía gástrica (úlcera péptica o tumores) o su función (hipomotilidad, distensión); anomalías de los órganos adyacentes (hernia hiatal, hernia diafragmática congénita, parálisis del nervio frénico, asplenismo y bazo errante), trauma o cirugía abdominal previa con sección de ligamentos gástricos, como en el trasplante hepático y la funduplicatura. ${ }^{7,9}$

Su presentación clínica varía según la etiología, velocidad de progresión, tipo de vólvulo, el grado de rotación y obstrucción resultante, por lo que los síntomas pueden imitar cualquier cuadro abdominal. En la forma aguda, la tríada de Borchardt (distensión epigástrica severa con dolor abdominal, arcadas intratables e incapacidad para pasar una SNG) es diagnóstica en 70\% de los pacientes adultos. ${ }^{3,10}$ El subagudo se caracteriza por malestar abdominal vago, mientras que en el VG crónico su sintomatología es inespecífica con dolor epigástrico, saciedad precoz, náuseas o vómito, ${ }^{8}$ otros síntomas pueden ser dolor torácico atípico, anemia, pérdida de peso, disnea, reflujo, distensión abdominal postprandial o disfagia y pueden aparecer de forma irregular durante semanas o años. ${ }^{7}$ Se debe enfatizar en la alta probabilidad de agudización del VG crónico. Las complicaciones secundarias de VG agudo son el íleo gástrico, isquemia pilórica, necrosis gástrica con perforación e incluso muerte. ${ }^{3,11} \mathrm{El}$ diagnóstico suele ser difícil por la poca sospecha clínica, puede ir desde ser un hallazgo radiológico incidental hasta situaciones urgentes. ${ }^{12}$ En el examen con rayos $\mathrm{X}$ se puede detectar la dilatación gástrica con escasez de gas en la parte restante del intestino. ${ }^{2} \mathrm{Si}$ el VG es secundario a un defecto diafragmático, el aire retrocardiaco se puede encontrar un nivel de burbuja o aire en el pecho (Figura 3), en especial en la forma mesentérico- 
axial la sombra gástrica muestra doble nivel de aire y fluido en bipedestación, mientras que en el órgano-axial, el estómago se coloca más horizontalmente con un nivel de fluido único. ${ }^{13} \mathrm{La}$ serie gastrointestinal superior se considera más específica que la radiografía, pues revela obstrucción del estómago en el sitio del vólvulo y su distensión a nivel del diafragma (Figura 4). ${ }^{8}$ En este caso, la presentación no fue la clásica, por lo cual no se logró un diagnóstico certero preoperatorio. Fue necesario realizar una tomografía axial computada (TAC), lo cual concuerda con el estudio de Mazaheri y colaboradores, donde se apoya su uso como el estudio de mayor sensibilidad y especificidad para el diagnóstico de VG, con una precisión de 90\%, el hallazgo más importante es el punto de transición del pico pilórico, ${ }^{14}$ confirma el diagnóstico con detalles anatómicos y posibles condiciones asociadas (hernias paraesofágicas, diafragmáticas, eventración diafragmática). En la etapa tardía del VG, el compromiso vascular puede resultar en hallazgos de isquemia gástrica, ulceración o fisuras de la mucosa gástrica. ${ }^{11,15}$ El estado hemodinámico de nuestra paciente no permitió que se realizaran más estudios y se prosiguió con la laparotomía exploradora. El tratamiento puede ser conservador o quirúrgico dependiendo de la presentación clínica y de
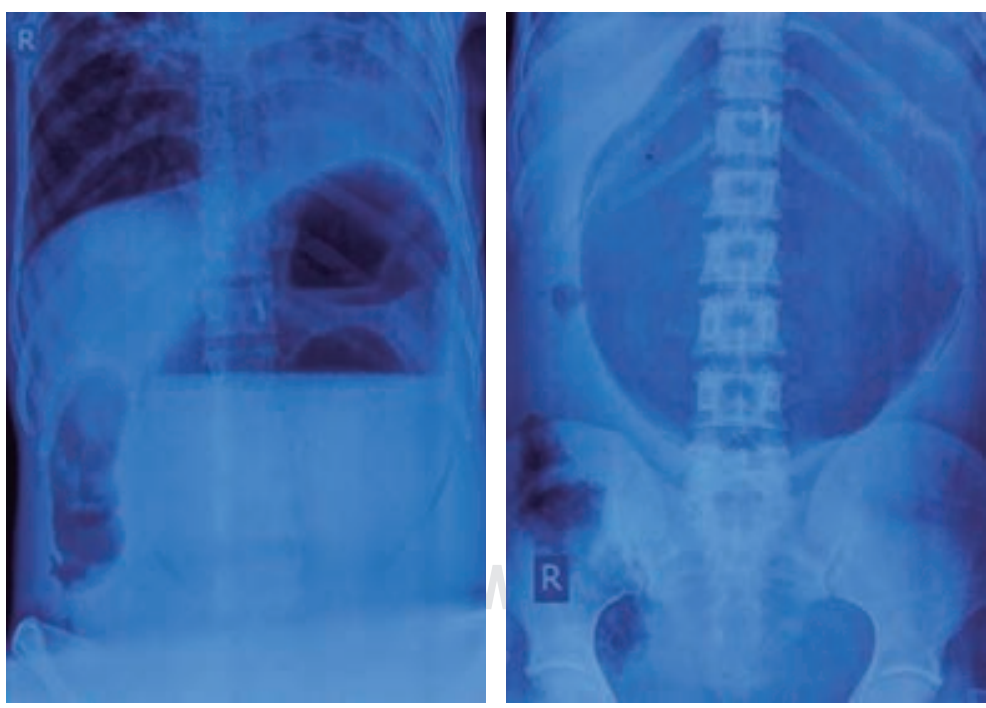

Figura 3: Radiografía de paciente con vólvulo gástrico donde el estómago distendido ocupa casi todo el abdomen, predominante en epigastrio y región umbilical, además de un gran nivel líquido en la imagen de la izquierda. Tomado de: Kumar $B$, et al. ${ }^{16}$

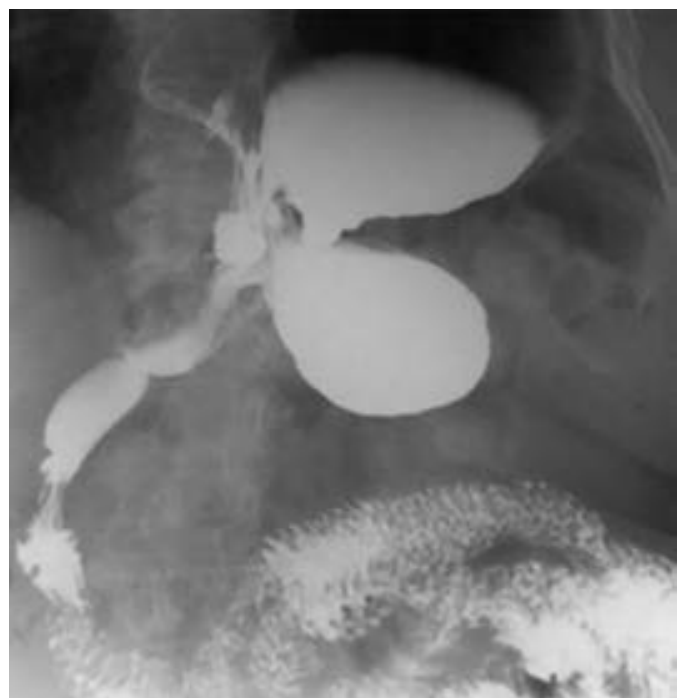

Figura 4: Estudio baritado: se observa hernia paraesofágica con cuerpo gástrico en cavidad torácica.

las posibles anomalías asociadas. Mientras que en fase aguda debe tratarse con intervención quirúrgica de emergencia, no hay pautas claras sobre el manejo del VG crónico. ${ }^{11,14}$ El manejo inicial debe ser con descompresión con SNG, seguida de cirugía para comprobar la viabilidad gástrica, resecar si hay necrosis y realizar tratamiento quirúrgico definitivo: reducción de la rotación, gastrostomía, gastropexia y reparación de los defectos predisponentes. ${ }^{17}$ En un estudio retrospectivo sobre pacientes con VG y manejo conservador a cinco años, se encontró que la recurrencia sintomática fue de $64 \%$, pero ésta sólo es una opción para los pacientes con VG crónico, en especial los mayores de 60 años y con alto riesgo quirúrgico, ${ }^{17}$ implica reducción o gastrostomía endoscópica percutánea más tratamiento procinético y antisecretor; sin embargo, existe un riesgo alto de perforación gástrica. ${ }^{16} \mathrm{En}$ el caso presentado, se determinó que la paciente no era candidata para este manejo. ${ }^{1,18}$ La cirugía mínimamente invasiva, como la desrotación endoscópica y la cirugía laparoscópica de una sola incisión, ha ganado terreno sobre las técnicas clásicas debido a la menor tasa de complicaciones, de sangrado y de estancia hospitalaria, por eso es necesario establecer un procedimiento estándar bajo esta técnica. Habría que considerarse desde el inicio la opción de un manejo más drástico con la realización de una gastrectomía total con 
anastomosis esofagoyeyunal término-terminal, tratando de evitar las complicaciones, el riesgo de infección y el trauma quirúrgico repetitivo. ${ }^{12}$

\section{CONCLUSIONES}

A pesar del manejo integral del caso, su desenlace fue fatal debido al tiempo de evolución y las comorbilidades propias de la paciente. Esto nos exige analizar y hacer énfasis sobre las opciones y cuidados para mejorar condiciones pre y postoperatorias de los casos con esta entidad. El VG representa un reto para el cual se debe tener una alta sospecha diagnóstica ante el abordaje de un abdomen agudo y apoyarse en estudios de imagen con alta sensibilidad y especificidad para llegar a un diagnóstico acertado, por tanto, es necesario mantener una constante actualización y entrenamiento para hacer frente a este tipo de patologías.

\section{REFERENCIAS}

1. Takahashi T, Yamoto M, Nomura A, Ooyama K, Sekioka A, Yamada Y, et al. Single-incision laparoscopic gastropexy for mesentero-axial gastric volvulus. Surg Case Rep. 2019; 5: 19.

2. Akhtar A, Siddiqui FS, Sheikh AAE, Sheikh AB, Perisetti A. Gastric volvulus: a rare entity case report and literature review. Cureus. 2018; 10: e2312.

3. Caldaro T, Torroni F, Romeo E, Federici di Abriola G, Dall'Oglio L. Gastric volvulus. In: Till H, Thompson M, Foker J, Holcomb G, Khan K. Esophageal and gastric disorders in infancy and childhood. Berlin, Heidelberg: Springer; 2017. Vol. 1. pp. 1355-1360.

4. Chand K, Dey SK, Shaw SC. Chronic gastric volvulus: Cause of feed intolerance. Indian J Pediatr. 2018; 85: 686-687.

5. Murata R, Kamiizumi Y, Ishizuka C, Kashiwakura $\mathrm{S}$, Tsuji T, Kasai H, et al. Recurrent gastric volvulus associated with a gastrointestinal stromal tumor: A case report. Int J Surg Case Rep. 2019; 57: 126-129.

6. Rajkumar JS, Venkatesan G, Rajkumar A, Prabhakaran R, Akbar S. A case of organo-axial gastric volvulus following laparoscopic fundoplication: a case report. Indian J Surg. 2017; 79: 357-359. doi: 10.1007/s12262-016-1584-9.

7. Al Daoud F, Daswani GS, Perinjelil V, Nigam T. Acute organoaxial gastric volvulus: a massive problem with a twist-case report. Int J Surg Case Rep. 2017; 41: 366-369.

8. Ostiz Llanos M, Ruiz Goikoetxea M, Cozcolluela C. Vólvulo gástrico crónico: una causa infrecuente de dispepsia. RAPD online. 2018; 41: 311-313.

9. Moreno-Egea A, Morales Cuenca G. Vólvulo gástrico por hernia diafragmática crónica. Rev Chil Cir. 2016 68: 205-207.

10. Moore C, Matthews LR, Danner O, Taha A, BashanGilzenrat A, Nguyen J, et al. "Black esophagus” and gastric volvulus following slipped laparoscopic adjustable gastric band. Obes Surg. 2018; 28: 2941-2948.

11. Sánchez CS, Vique BL, Ardiles CO, Herquiñigo RD. Vólvulo gástrico: ¿Por qué recordarlo? Revisión a propósito de un caso. Rev Chil Radiol. 2012; 18: 129-135.

12. Jain $R$, Singh $V$, Shah $U$. Total gangrenous mesentroaxial gastric volvulus: case report. Indian J Surg. 2018; 80: 616-617.

13. Eshiba A, Kotb M, Shehata S. Acute gastric volvulus through an unsuspected diaphragmatic hernia. J Pediatr Surg Case Rep. 2019; 41: 21-23.

14. Mazaheri P, Ballard DH, Neal KA, Raptis DA, Shetty AS, Raptis CA, et al. CT of gastric volvulus: interobserver reliability, radiologists' accuracy, and imaging findings. AJR Am J Roentgenol. 2019; 212: 103-108.

15. Ramos GP, Majumder S, Ravi K, Sweetser S. Role of diagnostic preoperative upper gastrointestinal endoscopy in radiologically confirmed gastric volvulus. Dig Dis Sci. 2018; 63: 3091-3096.

16. Kumar B, Kalra T, Namdeo R, Soni RK, Sinha A. Acute gastric volvulus: A vicious twist of tummy-case report. Int J Surg Case Rep. 2017; 30: 81-85.

17. Hsu YC, Perng CL, Chen CK, Tsai JJ, Lin HJ. Conservative management of chronic gastric volvulus: 44 cases over 5 years. World J Gastroenterol. 2010; 16: 4200-4205.

18. Yates RB, Hinojosa MW, Wright AS, Pellegrini CA, Oelschlager BK. Laparoscopic gastropexy relieves symptoms of obstructed gastric volvulus in highoperative risk patients. Am J Surg. 2015; 209 (5): 875-880.

\section{Consideraciones y responsabilidad ética}

Protección de personas y animales: Los autores declaran que para esta investigación no se han realizado experimentos en seres humanos ni en animales. Confidencialidad de los datos: Los autores declaran que han seguido los protocolos de su centro de trabajo sobre la publicación de datos de pacientes. Derecho a la privacidad y consentimiento informado: Los autores declaran que en este artículo no aparecen datos de pacientes.

Conflicto de intereses: No existe conflicto de intereses por parte de los autores.

Fuentes de financiamiento: Los autores no recibieron apoyo financiero por parte del Hospital Central del Estado de Chihuahua, durante la investigación, autoría y publicación del presente reporte.

Correspondencia:

Luis Bernardo Enríquez Sánchez

Departamento de Cirugía General del Hospital Central del Estado de Chihuahua. Calle Rosales, Núm. 3302, Roma Sur, 31350, Chihuahua, México.

Teléfono: (614) 180-0800

E-mail: investigationhcu@gmail.com 